\title{
Augmented serotonin content in density separated platelets of fibromyalgia patients
}

\author{
Milovanovic $\mathrm{M}^{1 *}$, Nilsson $\mathrm{S}^{2}$, Harakka PI ${ }^{3}$ and Gerdle $\mathrm{B}^{4}$ \\ ${ }^{1}$ Department of Social and Welfare, Linköping University, Linköping, Sweden. Department of Internal Medicine, Vrinnevi Hospital, Norrköping, Sweden \\ ${ }^{2}$ Primary Health Care and Department of Medical and Health Sciences, Linköping University, Linköping, Sweden \\ ${ }^{3}$ Department of Neurobiology, Society and Caring Sciences, Karolinska Institutet, Stockholm, Sweden \\ ${ }^{4}$ Division of Community Medicine, Department of Medical and Health Sciences, Faculty of Medicine and Health Sciences, Linköping University, Pain and \\ Rehabilitation Center, Anaesthetics, Operations and Specialty Surgery Center, Region Östergötland, Linköping, Sweden
}

\begin{abstract}
Introduction: Fibromyalgia syndrome (FMS) is a musculoskeletal pain and fatigue disorder with diffuse myalgia and lowered pain thresholds. Platelets they take up and store serotonin $(5-\mathrm{HT})$ and when activated they release 5-HT. 5-HT re-uptake inhibitors prevent 5-HT uptake by the synapses, but these drugs are clinically less effective in FMS. The current study examined 5-HT content in density separated platelets.

Material and methods: The study involves 20 female's patients (age $39 \pm 8$ (SD) years) with FMS. 16 females (65 \pm 8 years (SD) years) without FMS served as controls. The platelet population was separated according to density with a linear Percoll ${ }^{\mathrm{TM}}$ into 17 different density fractions. In all fractions, platelet counts was analyzed and determination of 5-HT content.

Results: The two study groups did not vary considerably concerning platelet distribution in the fractions. In contrast, normal and low-density platelets of FMS demonstrated higher serotonin content $(\mathrm{p}<0.05)$.

Discussion: The study shows that platelets of FMS contain more serotonin. It is possible that platelets have additional storage locations of serotonin beside dense granules. The results may indicate that an abnormal sensitivity to pain may be due to an instability in the 5-HT system in FMS. Serotonin inhibitors, often given to FMS obstructs serotonin uptake in the synapses. It is therefore tenable that neurons eventually release a higher amount of serotonin. The circulating serotonin is taken up by platelets. As a result, there are to be a reduced effectiveness for these drugs in FMS, and patient will still encounter pain difficulties.
\end{abstract}

\section{Introduction}

\section{Fibromyalgia syndrome (FMS)}

FMS is considered as a persistent pain disease. FMS is more than often connected with extensive pain, several tender points, and low energy [1]. The victims are mostly women and occurs approximately $2 \%$ of the general population [2]. Anxiety, depressive symptoms and major depression and are a feature of the disease [3]. The origin of and pathophysiology of FMS is still uncertain. Pathophysiological theories contain modifications in detailed neurotransmitters such as serotonin (5-HT). A likely involvement of 5-HT in FMS could therefore be possible. Some evidence suggest the hypothesis that an insufficiency in serotonergic neuronal operation could be linked to the pathophysiology of FMS $[4,5]$. Low concentrations of 5-HT in cerebrospinal fluid and in serum have been discovered in FMS. It is hypothesized to have a meaning in the pain threshold [6].

\section{Serotonin (5-HT)}

It is well established that 5-HT is important in human physiology. Nearly, $90 \%$ of the body 5 -HT substance is located in the upper gastrointestinal tract. The majority of circulating $5-\mathrm{HT}$ is metabolized in the liver $[7,8]$. 5-HT has a crucial role in synaptic activity additionally [9]. It influence cognitive functions for instance memory and learning [10]. Serotonergic neurons produce $5-\mathrm{HT}$ in the central nervous system. Almost all brain areas express 5-HT receptors [10]. Platelets do not produce 5-HT [11] but they gather the molecule in a way similar to serotonergic neurons $[12,13]$. The process needs energy $[14,15]$. When activated $5-\mathrm{HT}$ is released from platelet dense granules [11]. 5-HT2 receptors mediate the effect of 5-HT upon platelets [16]. 5-HT is a weak platelet agonist and does not activate platelets per se [17]. The activity of 5-HT is guarded by a particular 5-HT transporter (SETR), which arbitrates the intracellular reuptake of 5-HT. SETR are able to be blocked by discriminating 5 -HT reuptake inhibitors (SSRIs). It is well known that platelets, central or peripheral serotonergic neurons and intestinal epithelial cells express SETR. Common molecular and physiological features are shared in these locations $[18,19]$. Opinion is divided whether SSRIs are effective or not at FMS. Nociception refers to the physiological process of transmitting a painful stimulus from the periphery through afferent neurons to the cerebral cortex. It has been hypothesized that serotonergic neurotransmission has an important

Correspondence to: Micha Milovanovic, $\mathrm{PhD}$, Department of welfare and care, Faculty of Medicine and Health Sciences, Linköping University, SE-601 74, Norrköping, Sweden; Tel: +46700896320; E-mail: micha.milovanovic@liu.se

Key words: fibromyalgia, platelets, platelet heterogeneity, serotonin

Received: August 02, 2016; Accepted: August 24, 2016; Published: August 28, 2016 